Running head: The impact of facial age on sex perception

\title{
Aging affects sex categorization of male and female faces in opposite
}

\section{ways}

Nadine Kloth ${ }^{\mathrm{a}, \mathrm{b}}$, Madeleine Damm ${ }^{\mathrm{c}}$, Stefan R. Schweinberger ${ }^{\mathrm{a}, \mathrm{b}, \mathrm{c}}$, \& Holger Wiese $e^{\text {b,d }}$

${ }^{\mathrm{a}} \mathrm{ARC}$ Centre of Excellence in Cognition and its Disorders, School of Psychology, The University of Western Australia, 35 Stirling Highway, Crawley WA 6009, Australia

${ }^{b}$ DFG Research Unit Person Perception, Friedrich Schiller University of Jena, Leutragraben 1, 07743 Jena, Germany

${ }^{\mathrm{c}}$ Department of Psychology, Friedrich Schiller University of Jena, Am Steiger 3/1, 07743 Jena, Germany

${ }^{\mathrm{d}}$ Department of Psychology, Durham University, Queen’s Campus, E007

Wolfson Building, Stockton on Tees, TS17 6BH, United Kingdom

Please send correspondence to: Nadine Kloth, The University of Western Australia, School of Psychology (M304), 35 Stirling Highway, Crawley WA 6009, Australia, E: nadine.kloth@uwa.edu.au, P: +61-8-6488-3240

\section{Contact information of co-authors:}

Madeleine Damm, E: dammmadeleine@aol.com

Stefan R. Schweinberger, E: stefan.schweinberger@uni-jena.de, P: $+49-3641-945181$

Holger Wiese, E: holger.wiese@durham.ac.uk, P: +44 1913340433 


\begin{abstract}
Faces are rich in social information; they easily give away a person's sex, approximate age, feelings, or focus of attention. Past research has mostly focused on investigating the distinct facial signals and perceptual mechanisms that allow us to categorize faces on these individual dimensions. It is less well understood how the different kinds of facial information interact. Here we investigated how the age of a face affects the ease with which young and older adults categorize its sex. Disconfirming everyday intuition, we showed that sex categorization is not generally hampered for older faces. Although categorization of female faces took progressively more time with increasing age, the opposite was found for male faces (Experiment 1). Differential effects of stimulus blurring and inversion for male and female faces of different ages (Experiment 2) strongly suggest one feature as a crucial mediator of the interdependence of age and sex perception - skin texture.
\end{abstract}

Keywords: face perception; social perception; categorization; aging; skin texture 


\section{Introduction}

We can tell whether a face is male or female within fractions of a second (Bruce et al., 1993; O’Toole, Peterson, \& Deffenbacher, 1996). Various sources of information within the face allow us to make this decision with such efficiency. These include single facial features such as the eyes, nose, mouth, and chin (Brown \& Perrett, 1993; Bruce et al., 1993; Roberts \& Bruce, 1988) as well as the eyebrows and face outline (Yamaguchi, Hirukawa, \& Kanazawa, 1995). We also base sex categorizations on configural information, i.e., relational information between facial features, such as the distance between the eyelids and the brows (Campbell, Wallace, \& Benson, 1996). The importance of such configural information for sex decisions is reflected in the fact that face inversion, a manipulation that is thought to disturb configural processing, substantially impairs sex classification (Bruce et al., 1993; Wiese, Kloth, Güllmar, Reichenbach, \& Schweinberger, 2012).

Here we ask whether other social signals, specifically the age of a face, also affect our perception of its sex. To date there is a remarkable lack of research on the effect of variations in facial age on the perception of sex in faces, with only very few exceptions (Johnston, Kanazawa, Kato, \& Oda, 1997; Quinn \& Macrae, 2005). The virtual absence of such research perhaps relates to the common assumption that invariant facial signals are processed rather independently, and that different facial categorizations rely on distinct visual features (Bruce \& Young, 1986). However, some facial characteristics might be informative for multiple categorizations of faces, and the change of one facial aspect might therefore also affect the perception of the face on a different dimension (e.g., Adams \& Kleck, 2003; Schweinberger \& Soukup, 1998). 
There is indeed evidence to support the idea that the perception of sex at least partly relies on information that also allows for age categorization. Wiese, Schweinberger, and Neumann (2008) found that participants were faster to categorize the age of faces that had earlier been categorized according to their sex, compared to new faces. This finding suggests that visual information underlying the perception of facial age had already been accessed during prior exposure, even when the task required a sex categorization.

Considering that sex and age categorizations of faces at least partly rely on shared information, it is possible that variations in age also affect the perception of sex from faces. However, the specific nature of such a relationship is as yet unspecified. A common everyday intuition seems to be that sex categorization becomes increasingly difficult as faces grow older. Strikingly, there is only very little empirical support for this assumption, with the exception of a single study. Quinn and Macrae (2005) investigated whether perceivers simultaneously attend to a person's various “identities" (e.g., as a woman or a senior citizen). They asked participants to categorize faces according to their sex, while either presenting young and old faces within each experimental block or keeping age constant. In blocks in which faces varied in both age and sex, Quinn and Macrae found that participants were faster to categorize the sex of young faces (20 to 30 years old) than of older faces ( $>60$ years old). The authors concluded that this age-dependent sex categorization "may reflect the fact that facial changes during aging tend to minimize apparent sex differences between female and male faces“ (p. 473).

Quinn and Macrae's (2005) interpretation is intuitively convincing, and it is also concordant with changes in sex hormone levels during adulthood. Both 
testosterone and estrogen reach peak levels in adolescence and early adulthood, causing sexual dimorphism in face and body appearance. As the levels of these hormones start to decrease in a person's forties to fifties (Feldman et al., 2002; Lamberts, van den Beld, \& van der Lely, 1997), sexual dimorphism in faces might also be expected to decrease in older age.

However, data from another study raise the interesting possibility that face age might affect the perception of sex from male and female faces differently. Johnston, Kanazawa, Kato, \& Oda (1997) measured participants' reaction times when categorizing male and female child and adult faces according to sex and age (in separate blocks). During sex categorizations, participants responded faster to adult than child faces, a finding that is unsurprising, given that sexual dimorphism in the face is more pronounced after puberty than before. More importantly, however, when categorizing the faces for age (young vs. old), participants responded more slowly to female adult faces than to any other face condition, i.e., male adult and children's faces. This finding suggests that female adult faces are more difficult to distinguish from children's faces than male adult faces.

We find this aspect of Johnston et al.'s data particularly interesting because it might indicate an important role of skin texture in any potential interaction of face age and face sex. Here, we use the term "skin texture" to refer to the detailed pattern of the skin surface, as determined by the presence or absence of lines, wrinkles, visible pores, and stubble ${ }^{1}$. The skin texture of young adult fe-

${ }^{1}$ Note that this definition deviates from a less specific use of the term texture (often used interchangeably with the term reflectance), as a general measure of the reflecting properties of the skin surface. 
male faces is smoother than that of male faces and therefore more similar to that of children, which possibly delays age discrimination of these faces when presented amongst children's faces. Critically, skin texture is not only a reliable cue to age (George \& Hole, 1995; 2000; Lai, Oruç, \& Barton, 2013; for a review, see Rhodes, 2009), but is also relevant for sex decisions: Bruce et al. (1993) demonstrated that sex perception is substantially impaired relative to baseline performance when participants are asked to categorize laser-scanned face stimuli, which are lacking texture information.

The idea that the age and sex of faces might be processed interactively, possibly moderated by a shared reliance on skin texture information, is also in line with the finding that feminized faces are perceived to be younger than masculinized faces (Perrett et al., 1998). Moreover, the age of adult female faces tends to be underestimated, whereas the age of male faces tends to be slightly overestimated (Voelkle, Ebner, Lindenberger, \& Riediger, 2012, a pattern that appears to be reversed during adolescence when girls are perceived to be olderlooking than boys of the same age, Willner \& Rowe, 2001).

Importantly, if smooth skin is indicative of both youth and femininity, age-related changes in skin texture would be predicted to not only influence our perception of facial age itself, but to also hamper sex categorization of older female faces more than that of male faces. Here, we systematically investigated the effect of face age on sex perception in adult faces. Our aim was to establish whether increasing age affects the efficiency of sex classifications of male and female faces similarly, in line with previous conclusions (Quinn \& Macrae, 2005), or whether the perception of sex in female and male faces is differentially 
affected by aging, as would be predicted based on the importance of skin texture information for both age and sex classifications (Bruce et al., 1993; Lai, Oruç, \& Barton, 2013). To this end, we asked participants to categorize the sex of male and female faces from three different age groups, ranging from young adulthood to older age. Based on the fact that a reduction of collagen, elastin and subcutaneous adipose tissue makes aging skin gradually lose its smooth texture, which serves as an indicator of both youth and femininity, we predicted differential effects of age on sex categorization for male and female faces. Specifically, female faces should take longer to categorize with increasing age. In contrast, no such detrimental effects were predicted for male faces. In fact, if the absence of smooth skin texture actually serves as a signal for masculinity, male faces might even be categorized more easily with increasing age (cf., Voelkle et al., 2012).

\section{Experiment 1}

\subsection{Materials and Methods}

\subsubsection{Participants}

Twenty young adults $(9$ men, age range $18-30$ years, $M=24.5, S D=$ 3.5) participated in the experiment. All participants were naïve to the purpose of the study and had normal or corrected-to-normal vision. The study was in accordance with the ethical guidelines of the Declaration of Helsinki. Written informed consent was obtained from all participants before the experiment.

\subsubsection{Stimuli}

Color pictures of 144 unfamiliar individuals were obtained from the FACES database of the Max Planck Institute for Human Development (Ebner, Riediger, \& Lindenberger, 2010). Faces were of three different age groups, each 
represented by 48 individuals ( $50 \%$ male). Young faces ranged from 19 to 28 years (male faces: $20-28$ years, $M=23.9$ years, female faces: $19-28$ years, $M=$ 22.5 years), middle-aged faces ranged from 43 to 55 years (male faces: 43-55 years, $M=49.0$, female faces: $45-55$ years, $M=48.9$ ), and old faces ranged from 69 to 78 years (male faces: $70-78$ years, $M=72.5$, female faces: $69-78$ years, $M$ $=73.1$ ). Within each age group, male and female faces did not significantly differ in age, all $t \mathrm{~s}<1.7$, all $p \mathrm{~s}>.10$.

Pictures did not contain gender-specific features such as beards, glasses, make-up, or jewelry and were edited so that the hair was removed from the image as completely as possible ${ }^{2}$. Stimuli measured $8.2 \mathrm{~cm}$ in width and $10.1 \mathrm{~cm}$ in height, corresponding to $5.2^{\circ} \times 6.4^{\circ}$ at a viewing distance of $90 \mathrm{~cm}$, which was kept constant using a chin rest.

Please insert Figure 1 about here

\subsubsection{Design and Procedure}

Face age (young, middle-aged, old) and Face sex (female, male), were varied within participants, resulting in six experimental conditions. Participant sex was considered as between-participants factor. In a dimly lit room, stimuli were presented in color on a computer monitor with a black background. Individual stimuli were presented twice in separate blocks in otherwise randomized order. Each face was presented for $1000 \mathrm{~ms}$ with an inter-stimulus interval of $1500 \mathrm{~ms}$, and participants were asked to indicate the sex of each face as quickly

${ }^{2}$ In a few cases, female faces had hairstyles that were impossible to completely edit out due to fringes covering parts of the forehead. However, this was a rare occurrence and evenly spread across all age groups. 
and accurately as possible by pressing one of two buttons. The assignment of keys to response categories was counterbalanced across participants. Overall, there were 288 trials in the experiment, 48 per condition. A self-paced break was inserted after the first half of the trials.

\subsection{Results and Discussion}

\subsubsection{Accuracies}

Proportions of correct responses were analyzed in a repeated-measures analysis of variance (ANOVA) with Face age (young, middle-aged, old) and Face sex (female, male) as within-participants factors and Participant sex (female, male) as between-participants factor. There were significant main effects of Face age, $F(2,36)=20.89, p<.001, \eta_{\mathrm{p}}{ }^{2}=.54$, and Face sex, $F(1,18)=$ 27.91, $p<.001, \eta_{\mathrm{p}}{ }^{2}=.61$, which were qualified by an interaction of Face age and Face sex, $F(2,36)=22.86, p<.001, \eta_{\mathrm{p}}^{2}=.56$.

Planned pairwise comparisons were conducted to follow up on the interaction, exploring effects of age on the accuracy of sex categorization for male and female faces separately. Sex categorization of female faces became less accurate with increasing age. There was a trend for participants to be more accurate at categorizing young than middle-aged female faces, $t(19)=1.92, p=.07$, $\mathrm{d}=0.43$, which in turn were categorized significantly more accurately than old female faces, $t(19)=6.07, p<.001, \mathrm{~d}=1.36$. For male faces, participants were significantly less accurate at categorizing young than middle-aged faces, $t(19)=$ 3.91, $p=.001, \mathrm{~d}=0.87$, whereas accuracies for middle-aged and old faces did not differ significantly, $t(19)=0.81, p=.43, \mathrm{~d}=0.18$. Comparing accuracies between the different sexes at each age level revealed that participants more accurately categorized female than male sex when faces were young, $t(19)=2.43$, 
$p=.025, \mathrm{~d}=0.54$, but were more accurate at categorizing male than female sex when faces were middle-aged, $t(19)=3.58, p=.002, \mathrm{~d}=0.80$, or old, $t(19)=$ $6.94, p<.001, \mathrm{~d}=1.55$ (Figure 2a).

There were no other significant effects, all $F \mathrm{~s}<1.6$, all $p \mathrm{~s}>.21$, all $\eta_{\mathrm{p}}{ }^{2} \mathrm{~s}$ $<.09$.

\subsubsection{Reaction times}

For each participant, trials with incorrect responses and trials with RTs more than $3 S D$ above or below their mean RT in each individual condition were excluded before statistical analysis (leading to an exclusion of $6 \%$ of the trials). Reaction times were then entered into an ANOVA with Face age (young, middle-aged, old) and Face sex (female, male) as within-participants factors and Participant sex (female, male) as between-participants factor. There was a significant main effect of Face sex, $F(1,18)=11.56, p=.003, \eta_{\mathrm{p}}{ }^{2}=.39$, which was qualified by a significant Face age $\mathrm{x}$ Face sex interaction, $F(2,36)=70.16, p<$ $.001, \eta_{\mathrm{p}}^{2}=.80$.

Planned comparisons revealed that sex categorization of female faces took longer as face age increased. Young female faces were categorized faster than middle-aged female faces, $t(19)=9.87, p<.001, \mathrm{~d}=2.20$, which in turn were categorized faster than old female faces, $t(19)=2.81, p=.011, \mathrm{~d}=0.63$. For male faces, this pattern was reversed. Young male faces were categorized significantly more slowly than middle-aged male faces, $t(19)=8.98, p<.001$, d $=2.01$, which in turn were categorized more slowly than old male faces, although this last comparison was not significant, $t(19)=1.81, p=.087, \mathrm{~d}=0.40$ (Figure 2b). 
No other effects were significant, all $F_{\mathrm{s}}<2$, all $p \mathrm{~s}>.15$, all $\eta_{\mathrm{p}}{ }^{2} \mathrm{~s}<.10^{3}$.

Please insert Figure 2 about here.

In short, Experiment 1 confirmed our prediction that participants would need increasingly more time to correctly categorize the sex of female faces as face age increased. We did not predict the same detrimental effect of increasing age on the categorization of sex in male faces. Instead, we reasoned that if the absence of smooth skin texture actually served as a signal for masculinity, male faces might even be categorized more easily with increasing age. This was indeed the case. We found that participants took less time to accurately categorize male faces the older they were. The pattern of effects observed in accuracy data ruled out that the observed reaction time effects were driven by speed-accuracy tradeoffs. Instead, participants did not only categorize female faces faster, they also responded more accurately the younger the faces were. For male faces, the pattern was reversed and participants responded faster, and more accurately, the older male faces were.

${ }^{3}$ Since there was no theoretical reason to predict different effects for the first and second block of the experiment, block was not considered as a factor in the main analysis. Instead, data were collapsed across experimental blocks to enhance statistical power. To confirm the appropriateness of this approach, we performed an additional analysis considering Block (first run of trials, second run of trials) as an additional within-participants factor. This ANOVA revealed a significant main effect of Block, $F(1,19)=$ $5.58, p=.029, \eta_{p}^{2}=.23$, indicating that participants responded faster in the second than the first block. The analysis further revealed a significant main effect of Face sex, $F(1,19)=10.40, p=.004, \eta_{p}^{2}=.35$, and a significant Face age $\times$ Face sex interaction, $F(2,38)=71.85, p<.001, \eta_{p}{ }^{2}=.79$. The interaction of Block, Face Age, and Face Sex was not significant, $F(2,38)=.07, p=.94, \eta_{p}^{2}=.003$, indicating that the observed pattern of opposite effects of age on sex categorization for male and female faces did not differ significantly between the experimental blocks. 


\section{Experiment 2}

The specific predictions for Experiment 1 directly followed from considerations of skin texture as an indicator of both face age and face sex. However, Experiment 1 did not directly examine the contribution of skin texture information to participants' performance in the sex categorization task. Experiment 2 was designed to establish this relationship more directly. To this end, we asked a new sample of participants to categorize young, middle-aged, and old male and female faces according to their sex. To directly probe the role of skin texture as a likely mediator of the effect of face age on sex categorizations, we presented both full-spectrum natural photographs and low-pass filtered versions as stimuli. Spatial frequency filtering is a common manipulation in face perception research that has been useful in demonstrating that low spatial frequencies are particularly important for (holistic) face perception, but that both low and high spatial frequencies are required for fast and accurate face detection (e.g., Goffaux \& Rossion, 2006; Halit, de Haan, Schyns, \& Johnson, 2006). Here, we used a relatively strong low-pass filter to substantially attenuate texture cues and establish their contribution to sex decisions in the different experimental conditions. We assumed that low-pass spatial filtering should affect participants' performance only very little in those experimental conditions, in which sex categorization relies particularly strongly on smooth skin texture, i.e., the relative absence of high spatial frequency information. Experiment 1 suggests that this will be the case for sex categorizations of female faces. In contrast, performance in those conditions in which participants base sex categorizations on less smooth skin texture, should be impeded for low-pass filtered stimuli. Experiment 1 suggests that this will be the case for sex categorizations of male faces, particularly of old age. 
In addition, we also sought to establish the experimental conditions in which participants were particularly reliant on configural processing when performing sex categorizations. As outlined in the introduction, configural face information, for instance the distance between the eyes and the eyebrows, can be informative for sex discrimination (Campbell, Wallace, \& Benson, 1996), and the processing of such information is disrupted when faces are inverted (e.g., Freire, Lee, \& Symons, 2000; Maurer, Le Grand, \& Mondloch, 2002). We therefore presented all stimuli in both upright and inverted orientation, and predicted that sex categorization performance should be particularly severely impeded by inversion in those experimental conditions with strong reliance on configural information.

Importantly, when considering the respective effects of spatial filtering and inversion on categorization performance, one should keep in mind that these are most likely interactive, rather than additive. Specifically, configural processing can be expected to become more important, the less an observer can rely on skin texture information. For instance, for old female faces, which lack a smooth skin texture that might indicate femininity, a more configural analysis style may become necessary to correctly identify their sex, making this experimental condition prone to relatively large inversion costs. Conversely, inversion can be expected to be less detrimental in experimental conditions, in which participants naturally rely more on skin texture, which can be easily sampled from both upside and inverted faces. Overall, we therefore predicted larger inversion costs for old female faces compared to younger female faces, for which sex categorizations can be more easily based on skin texture information alone. For male faces, the opposite pattern was expected, old male faces should be easily 
categorized as male based on skin texture alone, therefore, inversion was predicted to have less detrimental effects for old compared to younger male faces.

Finally, we tested both a young and an older group of participants. This decision was based on earlier research, which suggests that young and old observers process own-age and other-age faces differently (Fulton \& Bartlett, 1991, see Rhodes \& Anastasi, 2012, for a review), and that varying expertise with young and old faces might affect processes underlying categorization (e.g., Wiese, Schweinberger, \& Hansen, 2008). For instance, the difficulty to accurately categorize the sex of old female faces observed in Experiment 1 might be restricted to young participants, whereas older participants might exhibit no such difficulties due to their greater expertise with old faces.

\subsection{Materials and Methods}

\subsubsection{Participants}

Twenty new young adults (18 -28 years, $M=22.8,50 \%$ male $)$ and twenty older participants (62 - 75 years, $M=67.2,50 \%$ male) took part in Experiment 2. All participants were naïve to the purpose of the study and had normal or corrected-to-normal vision. For the older participants, this was confirmed using the "Freiburg Visual Acuity \& Contrast Test" (Bach, 1996). The study was in accordance with the ethical guidelines of the Declaration of Helsinki. Written informed consent was obtained from all participants before the experiment.

\subsubsection{Stimuli}

The stimulus set was based on the same 144 pictures used in Experiment 1. To directly explore the potential role of skin texture, additional low-pass filtered versions were created for each of the pictures (10 cycles/image). During 
the experiment, each of the stimuli was presented in both upright and inverted orientation (Figure 3).

Please insert Figure 3 about here.

\subsubsection{Design and Procedure}

Face age (young, middle-aged, old), Face sex (female, male), Filter setting (unblurred, blurred), and Orientation (upright, inverted) were varied within participants, resulting in 24 experimental conditions. Participant age group and Participant sex were considered as between-participants factors.

In a dimly lit room, stimuli were presented in color on a computer monitor with a black background. A constant viewing distance of $\sim 90 \mathrm{~cm}$ was ensured using a chinrest. Stimuli were presented twice in separate blocks, once in each block, for $1000 \mathrm{~ms}$ each in randomized order with an inter-stimulus interval of $1500 \mathrm{~ms}$. Participants were asked to indicate the sex of each face as quickly and accurately as possible by pressing one of two buttons. The assignment of keys to response categories was counterbalanced across participants. Overall, there were 1152 trials in the experiment, 48 per condition. Self-paced breaks were inserted after every 96 trials. The experiment took about 45 minutes.

\subsection{Results and Discussion}

Experiment 1 had revealed corresponding results for accuracies and reaction times, ruling out speed-accuracy tradeoffs as a potential origin for the opposite effect of face age on the speed with which participants categorize the sex of 
male and female faces. In the interest of brevity, we therefore only report the analysis of reaction times for Experiment $2^{4}$.

Before the main analysis, incorrect trials and trials with reaction times above or below $3 S D$ from the participant's mean RT in that condition were excluded ( $14.5 \%$ of all trials). Latencies of the remaining responses were analyzed in an ANOVA with Face age (young, middle-aged, old), Face sex (female, male), Filter setting (unblurred, blurred), and Orientation (upright, inverted) as within-participants factors and Participant age group (young, old) and Participant sex (female, male) as between-participants factors.

A main effect of Participant age group, $F(1,36)=22.95, p<.001, \eta_{\mathrm{p}}{ }^{2}=$ .39 , reflected faster reaction times of young $(M=632.6 \mathrm{~ms}, S E M=22.7)$ compared to older participants $(M=792.9 \mathrm{~ms}, S E M=22.7)$. Moreover, the analysis revealed a number of additional significant main effects and interactions. For the sake of brevity, most effects qualified by higher-order interactions will not be enumerated here.

\section{Opposite effects of age on sex categorization of female and male faces}

Most importantly, the ANOVA revealed a significant interaction of Face age and Face sex, $F(2,72)=212.64, p<.001, \eta_{\mathrm{p}}{ }^{2}=.86$. Replicating the results of Experiment 1, female faces took progressively longer to be correctly classified with increasing age $\left(M_{\text {young }}=677.3 \mathrm{~ms} \pm 19.9 S E M, M_{\text {midage }}=720.0 \mathrm{~ms} \pm\right.$ 20.6 SEM, $M_{\text {old }}=744.1 \mathrm{~ms} \pm 20.6$ SEM $), t(39)=11.95, p<.001, \mathrm{~d}=1.89$, and $t(39)=6.65, p<.001, \mathrm{~d}=1.05$, for the comparison of reaction times to young vs. middle-aged and middle-aged vs. old female faces, respectively. In contrast,

\footnotetext{
${ }^{4}$ We ran an additional analysis on accuracies, which confirmed the main results obtained for reaction times and ruled out speed-accuracy-tradeoffs.
} 
male faces were classified progressively faster with increasing age ( $M_{\text {young }}=$ $753.9 \mathrm{~ms} \pm 23.1 S E M, M_{\text {midage }}=698.0 \mathrm{~ms} \pm 22.0 S E M, M_{\text {old }}=683.0 \mathrm{~ms} \pm 22.0$ SEM), $t(39)=11.55, p<.001, d=1.83$, and $t(39)=5.02, p<.001, d=0.79$, for the comparison of reaction times to young vs. middle-aged and middle-aged vs. older male faces, respectively (Figure 4a).

\section{Please insert Figure 4 about here.}

\section{Effects of spatial filtering}

The main pattern of opposite effects of age on the latency of sex categorization of male and female faces was present for both unblurred and blurred faces (Figure 5a). A significant interaction of Facial age, Facial sex, and Filter setting, $F(2,72)=11.43, p<.001, \eta_{\mathrm{p}}{ }^{2}=.24$, reflected opposite effects of Filter settings on the latency of sex categorization of faces from the different age groups for male and female faces. For female faces, blurring did not significantly affect reaction times to young, $t(39)=1.75, p=.09, \mathrm{~d}=0.28$, or middle-aged faces, $t(39)=1.20, p=.28, \mathrm{~d}=0.19$. However, for old female faces, sex categorization was significantly faster in the blurred condition compared to the unblurred condition, $t(39)=2.10, p=.046, d=0.33$ (Figure 5a). For male faces, blurring tended to slow down sex decisions for young faces, $t(39)=2.01, p=$ $.052, \mathrm{~d}=0.32$, and significantly delayed sex categorization of middle-aged, $t(39)$ $=5.93, p<.001, \mathrm{~d}=0.94$, and old faces, $t(39)=7.96, p<.001, \mathrm{~d}=1.26$.

Please insert Figure 5 about here. 


\section{Effects of stimulus orientation}

The main pattern of opposite effects of face age on the latency of sex categorization of male and female faces was present in both the upright and inverted conditions (Figure 5b). The interaction of Facial age, Facial sex, and Orientation, $F(2,72)=39.78, p<.001, \eta_{\mathrm{p}}{ }^{2}=.53$, reflected different amounts of inversion costs for female and male faces of the different age groups. For female faces, inversion costs increased as face age increased, with significantly larger inversion costs for middle-aged than young female faces, $t(39)=5.75, p<001$, d $=0.91$, and a trend towards larger inversion costs for old than middle-aged female faces, $t(39)=1.83, p=.074, \mathrm{~d}=0.29$ (Figure $5 \mathrm{~b}$ ). By contrast, for male faces, inversion costs decreased with increasing face age, with significantly larger inversion costs for young than middle-aged faces, $t(39)=3.61, p=.001, \mathrm{~d}=$ 0.57 , and for middle-aged than old faces, $t(39)=6.78, p<.001, \mathrm{~d}=1.07$ (Figure $5 b)$.

\section{Role of participant age}

The opposite effects of age on the latency of sex categorization of male and female faces were found in both young (Figure 4b) and older participants (Figure 4c). A significant interaction of Face age, Face sex, and Participant age group, $F(2,72)=4.56, p=.01, \eta_{\mathrm{p}}{ }^{2}=0.11$, was driven by the fact that young participants classified middle-aged female faces significantly more slowly than male faces of the same age, $t(19)=3.98, p=.001, d=0.89$, whereas older participants showed no such difference in response times to middle-aged female and male faces, $t(19)=0.98, p=.34, d=.02$.

\section{Role of participant sex}


The ANOVA further revealed a significant interaction of Face Sex and Participant Sex, $F(1,36)=7.45, p=.01, \eta_{\mathrm{p}}^{2}=.17$. Participants tended to respond faster when categorizing faces of their own sex rather than the opposite sex, although the comparison between response times for female and male faces was not significant for male, $t(19)=1.97, p=.06, \mathrm{~d}=0.44$, or female participants, $t(19)=1.39, p=.18, \mathrm{~d}=0.31$, when tested separately. A significant threeway interaction of Face Sex, Orientation, and Participant Sex, $F(1,36)=11.48$, $p=.002, \eta_{\mathrm{p}}{ }^{2}=24$, reflected that male participants experienced smaller inversion costs for male than female faces, $t(19)=3.13, p=.005, \mathrm{~d}=0.70$. A corresponding pattern of smaller inversion costs for female than male faces in female participants did not reach statistical significance, $t(19)=1.76, p=.095, \mathrm{~d}=0.39$.

\section{General Discussion}

We present the first evidence that face age differentially affects the perception of sex in male and female adult faces. While categorization of female faces took progressively more time with increasing age, the exact opposite pattern was found for male faces. This effect, which we consistently found in two experiments, suggests that female faces are increasingly difficult to identify as female when they grow older, whereas male faces are more easily perceived as male with increasing age. Our data therefore establish substantial effects of age on sex processing throughout the adult life span. Critically, the systematic pattern of these effects contradicts the intuitive assumption that it is generally more difficult to discriminate sex in older faces than in young adult faces (cf., Quinn \& Macrae, 2005).

We propose that the effect of age on sex categorization highlights a prominent role of skin texture for sex discrimination. This claim is sup- 
ported by the specific pattern of effects induced by the spatial filtering applied in Experiment 2. Although quite a drastic image manipulation, lowpass filtering (and thus erasing high-spatial frequency information) did not slow down sex categorization of young and middle-aged female faces. To the contrary, low-pass filtering even speeded up sex categorization of old female faces relative to unfiltered stimuli. A strikingly different pattern emerged for male faces, for which sex categorization was significantly slowed down for low-pass filtered stimuli, particularly when these were middle-aged or old. This pattern suggests that low-pass filtering eliminated textural cues to masculinity, thus artificially creating an impression of skin smoothness that is typically indicative of femininity.

The inversion effects observed for male and female faces are also consistent with our interpretation of the specific role of skin texture for sex decisions. Skin texture can be sampled from a single region within the face and can therefore be considered a facial feature. Compared to the processing of configural face information, which requires the integration of spatial relations between features, isolated feature processing is known to be less disrupted by face inversion (Rossion, 2008). Consequently, smaller face inversion costs in some conditions relative to others can serve as an indicator of a comparatively larger reliance on local feature than configural information.

Here, we observed that for female faces inversion costs increased with increasing face age. The opposite was true for male faces, for which inversion costs decreased with increasing age. Just like the filter effects, this pattern suggests that the processing of sex from female faces relies 
less on skin texture information, and more on configural information, as face age increases. In contrast, sex perception from male faces seems to rely more on skin texture information, and less on configural information, the older the face gets.

The combined evidence from the filtering and inversion manipulations strongly suggests a prominent role of skin texture information for sex discrimination as the main factor driving the effects of face age on sex perception observed here. These findings extend earlier evidence which has demonstrated that the skin surface, including skin texture and reflectance information, also plays an important role in face recognition more generally (Russell, Biederman, Nederhouser, \& Sinha, 2007; Russell \& Sinha, 2007). Our finding is in line with our initial consideration that sex and age categorizations are partly based on overlapping sources of information. At the same time, our participants were able to categorize the sex of faces based on other sources of information when texture information was unavailable. This finding, together with evidence for asymmetrical priming effects between age and sex categorization tasks (Wiese, Schweinberger, \& Neumann, 2008) and stronger neural activation during sex categorization compared to age categorization (Wiese, Kloth, Güllmar, Reichenbach, \& Schweinberger, 2012), underlines that other sources of information used for sex categorizations are distinct from those serving age categorization.

The effect of age on sex perception in female and male faces is largely independent of observer age. However, we found some differences between participant age groups for middle-aged faces (Figure 4). These differences may suggest that the impact of facial age on sex perception 
shows some sensitivity to top-down influences. Specifically, femininity might be more closely associated with youth while masculinity might be more closely associated with maturity. Given that our older participants were clearly older than the middle-aged faces, they might have perceived these faces to be comparatively young. By contrast, young participants might have perceived the middle-aged faces to be comparatively old. Such differences in self-centered age categorization, or differential expertise with middle-aged faces in older and younger adults, might explain the small differences between participant age groups with respect to middleaged faces.

Participants' sex also seems to have affected how quickly they performed in the sex categorization task. In Experiment 2, male and female participants tended to categorize own-sex faces faster than opposite-sex faces. At first sight, this pattern is reminiscent of the so-called own-gender bias in face recognition memory, i.e., the finding that participants more accurately remember faces of their own sex compared to those of the opposite sex (Wolff, Kemter, Schweinberger, \& Wiese, 2014; Wright \& Sladden, 2003), although this memory bias has often been found in female participants only (Lewin \& Herlitz, 2002, for a recent review, see Herlitz \& Loven, 2013).

However, upon closer consideration, the trend towards faster sex categorizations for own-sex than other-sex faces contradicts typical findings of own-group bias studies in face recognition. For instance, the ownrace bias, i.e., better recognition for own-race than other-race faces (Meissner \& Brigham, 2001) is typically accompanied by faster categori- 
zation of other-race relative to own-race faces (Levin, 1996; Valentine \& Endo, 1992). This is the opposite of the own-sex categorization advantage we found here. However, considering that the present support for an ownsex classification advantage is limited to a trend in Experiment 2, and was absent in Experiment 1 (for which power might not have been sufficient to reveal participant sex effects), and in other research on the own-sex bias (Wolff, Kemter, Schweinberger, \& Wiese, 2014), it might be premature to discuss the potential implications of this finding in great detail at the present stage.

Whereas our data clearly support our predictions of a differential effect of age on sex categorization for male and female faces, it needs to be noted that these findings are at some variance with those of Quinn and Macrae (2005). Their participants were generally faster to categorize the sex of young than old faces, irrespective of face sex. Interestingly, and in line with our findings, a bar graph depicting the results of Quinn and Macrae (2005, Figure 4) suggests that the advantage for young over old faces was much stronger for female than male faces. However, the followup tests of their significant face age $\mathrm{x}$ face sex interaction focus on showing faster sex categorization for young female than young male faces (again in line with the results of our study).

Several stimulus-related factors might potentially contribute to the differences between our findings and those of Quinn and Macrae (2005). First, the old faces in our study had a mean age of over 72 years (ranging from 69 to 78 years) and were presumably older than the faces used by Quinn and Macrae, which were selected based on a criterion of being over 
60 years old (with no means and ranges reported). Quinn and Macrae's stimuli might therefore be closer to the age of our middle-aged age category, for which we also found less clear-cut effects than for old faces. Second, Quinn and Macrae's stimuli were converted to grayscale and therefore lacked color information, which is an important source of information for sex decisions (Hill, Bruce, \& Akamatsu, 1995). Finally, Quinn and Macrae (2005) do not specify whether or not their stimulus faces were presented with full hairstyles. The presence of hairstyles might have provided participants with additional strategies to determine the sex of faces, possibly explaining some variance between their results and those of the present study.

To summarize, we have shown that face age strongly affects sex categorization of adult faces, which is in line with a previous report (Quinn \& Macrae, 2005). Importantly, our data go beyond previous findings by showing that increasing age does not generally impair categorization efficiency for both sexes. Instead, a detrimental effect of age seems to be selective to sex processing in female faces. Strikingly, male faces are more quickly classified as male with increasing age, indicating that signals of masculinity become stronger with age. Systematic effects of spatial filtering and face inversion suggest that this specific influence of age on sex perception is largely driven by one feature - the skin texture of the face. 


\section{Acknowledgements}

This work was supported by the Australian Research Council Centre of Excellence in Cognition and its Disorders (project number CE110001021) and by research grants from the Deutsche Forschungsgemeinschaft to HW and SRS (Wi 3219/4-2, FOR 1097/2; Wi 3219/5-2). The authors thank Andrea Kowallik for help with stimulus editing and data collection and Maria Rohmann and Sina Schneider for their support during data collection. 


\section{References}

Adams, R. B., \& Kleck, R.E. (2003). Perceived gaze direction and the processing of facial displays of emotion. Psychological Science, 14, 644-647.

Bach, M. (1996). The Freiburg Visual Acuity Test - Automatic measurement of visual acuity. Optometry and Vision Science, 73, 49-53.

Brown, E., \& Perrett, D. I. (1993). What gives a face its gender. Perception, 22(7), 829-840.

Bruce, V., Burton, A. M., Hanna, E., Healey, P., Mason, O., Coombes, A., et al. (1993). Sex discrimination: how do we tell the difference between male and female faces? Perception, 22, 131-152.

Bruce, V., \& Young, A. (1986). Understanding face recognition. British Journal of Psychology, 77, 305-327.

Campbell, R., Wallace, S., \& Benson, P. J. (1996). Real men don’t look down: Direction of gaze affects sex decisions on faces. Visual Cognition, 3, 393412.

Ebner, N.C., Riediger, M., \& Lindenberger, U. (2010). FACES - A database of facial expressions in young, middle-aged and older women and men: Development and validation. Behavior Research Methods, 42, 351-362.

Feldman, H. A., Longcope, C., Derby, C. A., Johannes, C. B., Araujo, A. B., Coviello, A. D., et al. (2002). Age trends in the level of serum testosterone and other hormones in middle-aged men: Longitudinal results from the Massachusetts Male Aging Study. The Journal of Clinical Endocrinology \& Metabolism, 87, 589-598. 
Freire, A., Lee, K., \& Symons, L. A. (2000). The face-inversion effect as a deficit in the encoding of configural information: Direct evidence. Perception, 29, 159-170.

Fulton, A., \& Bartlett, J. C. (1991). Young and old faces in young and old heads: The factor of age in face recognition. Psychology and Aging, 6, 623630.

George, P. A., \& Hole, G. J. (1995). Factors influencing the accuracy of age estimates of unfamiliar faces. Perception, 24(9), 1059-1073.

George, P. A., \& Hole, G. J. (2000). The role of spatial and surface cues in the age-processing of unfamiliar faces. Visual Cognition, 7(4), 485-509.

Goffaux, V., \& Rossion, B. (2006). Faces are "spatial" - Holistic face perception is supported by low spatial frequencies. Journal of Experimental Psychology-Human Perception and Performance, 32(4), 1023-1039.

Halit, H., de Haan, M., Schyns, P. G., \& Johnson, M. H. (2006). Is highspatial frequency information used in the early stages of face detection? Brain Research, 1117, 154-161.

Herlitz, A., \& Loven, J. (2013). Sex differences and the own-gender bias in face recognition: A meta-analysis review. Visual Cognition, 21(9-10), 13061336.

Hill, H., Bruce, V., \& Akamatsu, S. (1995). Perceiving the sex and race of faces - the role of shape and color. Proceedings of the Royal Society B - Biological Sciences, 261(1362): 367-373.

Johnston, R. A., Kanazawa, M., Kato, T., \& Oda, M. (1997). Exploring the structure of multidimensional face-space: The effects of age and gender. Visual Cognition, 4, 39-57. 
Lai, M., Oruç, I., \& Barton, J. J. S. (2013). The role of skin texture and facial shape in representations of age and identity. Cortex, 48(1), 252-265.

Lamberts, S. W. J., van den Beld, A., \& van der Lely, A.-J. (1997). The endocrinology of aging. Science, 278, 419-424.

Levin, D. T. (1996). Classifying faces by race: The structure of face categories. Journal of Experimental Psychology: Learning, Memory, and Cognition, 22(6), 1364-1382.

Lewin, C., \& Herlitz, A. (2002). Sex differences in face recognitionwomen's faces make the difference. Brain and Cognition, 50, 121-128.

Maurer, D., Le Grand, R., \& Mondloch, C. J. (2002). The many faces of configural processing. Trends in Cognitive Sciences, 6(6), 255-260.

Meissner, C. A., \& Brigham, J. C. (2001). Thirty years of investigating the own-race bias in memory for faces: a meta-analytic review. Psychology, Public Policy, and Law, 7(1), 3-35.

O’Toole, A. J., Peterson, J., \& Deffenbacher, K. A. (1996). An “otherrace effect" for classifying faces by gender. Perception, 25, 669-676.

Perrett, D. I., Lee, K. J., Penton-Voak, I., Rowland, D., Yoshikawa, S., Burt, D. M., et al., (1998). Effects of sexual dimorphism on facial attractiveness, Nature, 394, 884-887.

Quinn, K. A., \& Macrae, C. N. (2005). Categorizing others: The dynamics of person construal. Journal of Personality and Social Psychology, 88(3), 467-479.

Rhodes, M. G. (2009). Age estimation of faces: A review. Applied Cognitive Psychology, 23(1). 1-12. 
Rhodes, M. G., \& Anastasi, J. S. (2012). The own-age bias in face recognition: A meta-analytic and theoretical review. Psychological Bulletin, 138, 146174.

Roberts, T., \& Bruce, V. (1988). Feature saliency in judging the sex and familiarity of faces. Perception, 17, 475-481.

Rossion, B. (2008). Picture-plane inversion leads to qualitative changes of face perception. Acta Psychologica, 128, 274-289.

Russell, R., Biederman, I., Nederhouser, M., \& Sinha, P. (2007). The utility of surface reflectance for the recognition of upright and inverted faces. Vision Research, 47(2), 157-165.

Russell, R., \& Sinha, P. (2007). Real-world recognition: The importance of surface reflectance properties. Perception, 36(9), 1368-1374.

Schweinberger, S.R., \& Soukup, G. R. (1998). Asymmetric relationships among perceptions of facial identity, emotion, and facial speech. Journal of Experimental Psychology: Human Perception and Performance, 24, 1748-1765.

Valentine, T. \& Endo, M. (1992). Towards an exemplar model of face processing: the effects of race and distinctiveness. Quarterly Journal of Experimental Psychology: Human Experimental Psychology, 44a, 671-703.

Voelkle, M. C., Ebner, N. C., Lindenberger, U., \& Riediger, M. (2012). Let me guess how old you are: Effects of age, gender, and facial expression on perceptions of age. Psychology and Aging, 27, 265-277.

Wiese, H., Kloth, N., Güllmar, D., Reichenbach, J. R., \& Schweinberger, S. R. (2012). Perceiving age and gender in unfamiliar faces: An fMRI study on face categorisation. Brain and Cognition, 78, 163-168. 
Wiese, H., Schweinberger, S.R., \& Hansen, K. (2008). The age of the beholder: ERP evidence of an own-age bias in face memory. Neuropsychologia, $46,2973-2985$.

Wiese, H., Schweinberger, S.R., \& Neumann, M.F. (2008). Perceiving age and gender in unfamiliar faces: Brain potential evidence for implicit and explicit person categorization. Psychophysiology, 45, 603-615.

Willner, P., \& Rowe, G., (2001). Alcohol servers' estimates of young people's ages. Drugs: Education, Prevention and Policy, 8(4) 375-383.

Wolff, N., Kemter, K., Schweinberger, S. R., \& Wiese, H. (2014). What drives social in-group biases in face recognition memory? ERP evidence from the own-gender bias. Social, Cognitive, and Affective Neuroscience, 9, 580-590.

Wright, D. B., \& Sladden, B. (2003). An own gender bias and the importance of hair in face recognition. Acta Psychologica, 114, 101-114.

Yamaguchi, M. K., Hirukawa, T., \& Kanazawa, S. (1995). Judgment of gender through facial parts. Perception, 24(5), 563-575. 


\section{Figure 1}
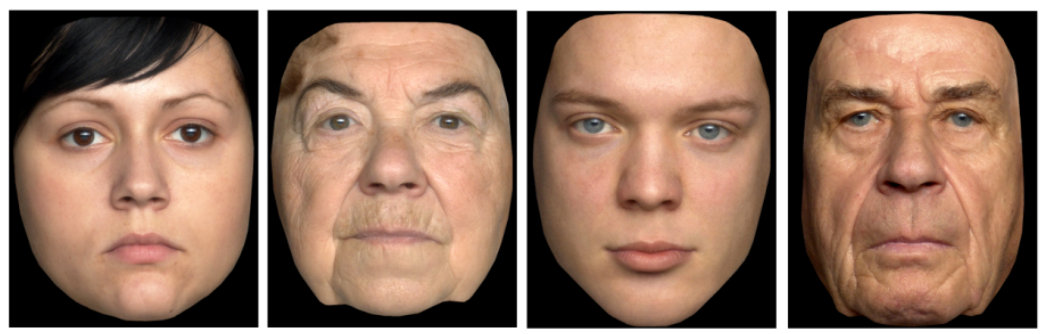

Figure 1: Examples of stimuli used in Experiment 1. From left to right: young female face, old female face, young male face, old male face. Please note that copyright limitations prevent depiction of stimulus examples for the middleaged category. 


\section{Figure 2}

a.

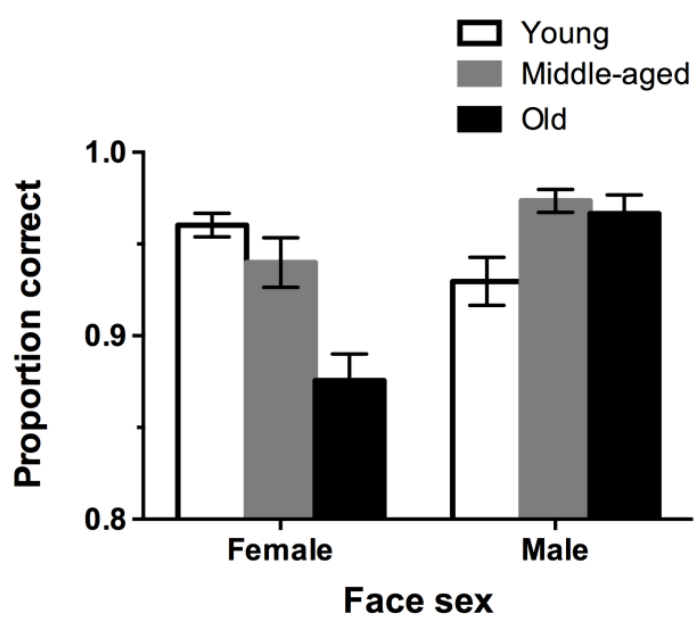

b.

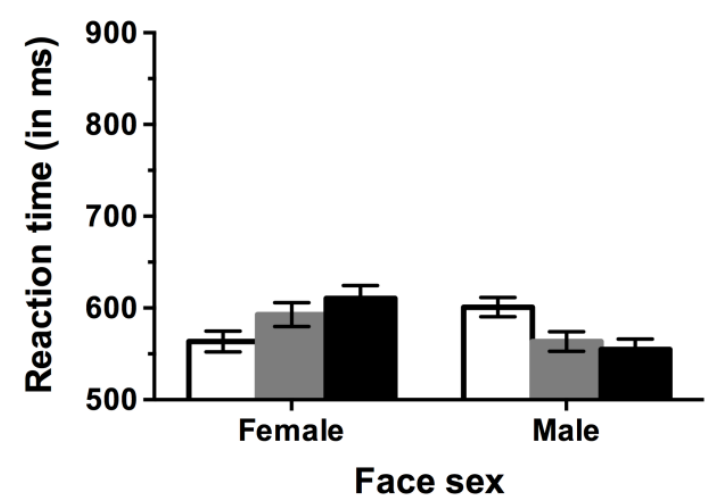

Figure 2: Results of Experiment 1. a. Mean proportion of correct sex categorizations depending on facial age for female and male faces as observed in Experiment 1. B. Mean response latencies of correct sex categorizations depending on facial age for female and male faces as observed in Experiment 1. Error bars indicate SEMs. 


\section{Figure 3}
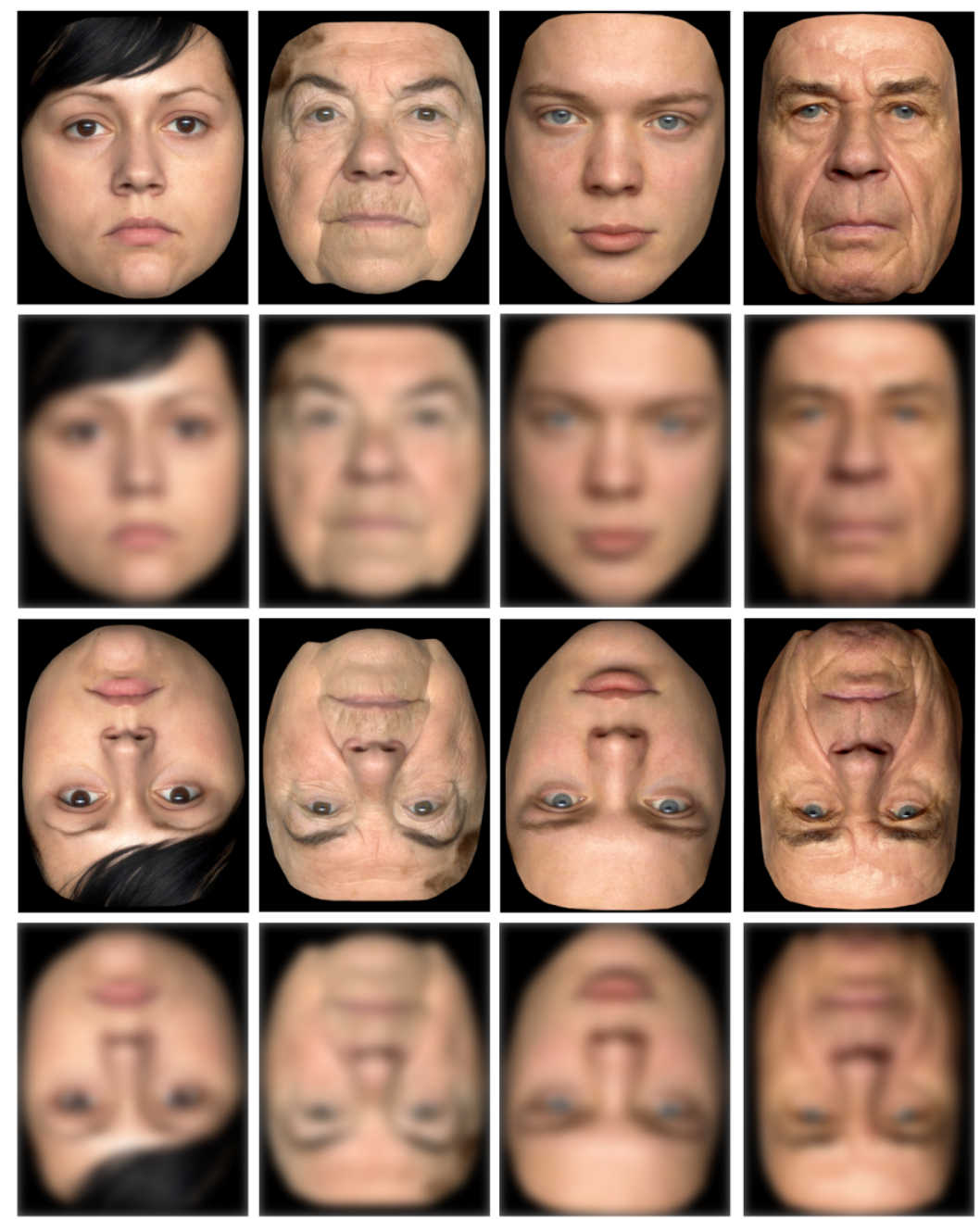

Figure 3 : Examples of stimuli used in Experiment 2. Columns (from left to right): young female face, old female face, young male face, old male face.

Rows (from top to bottom) : upright unfiltered version, upright filtered version, inverted unfiltered version, inverted filtered version. Please note that copyright limitations prevent depiction of stimulus examples for the middle-aged category. 
Figure 4

b.
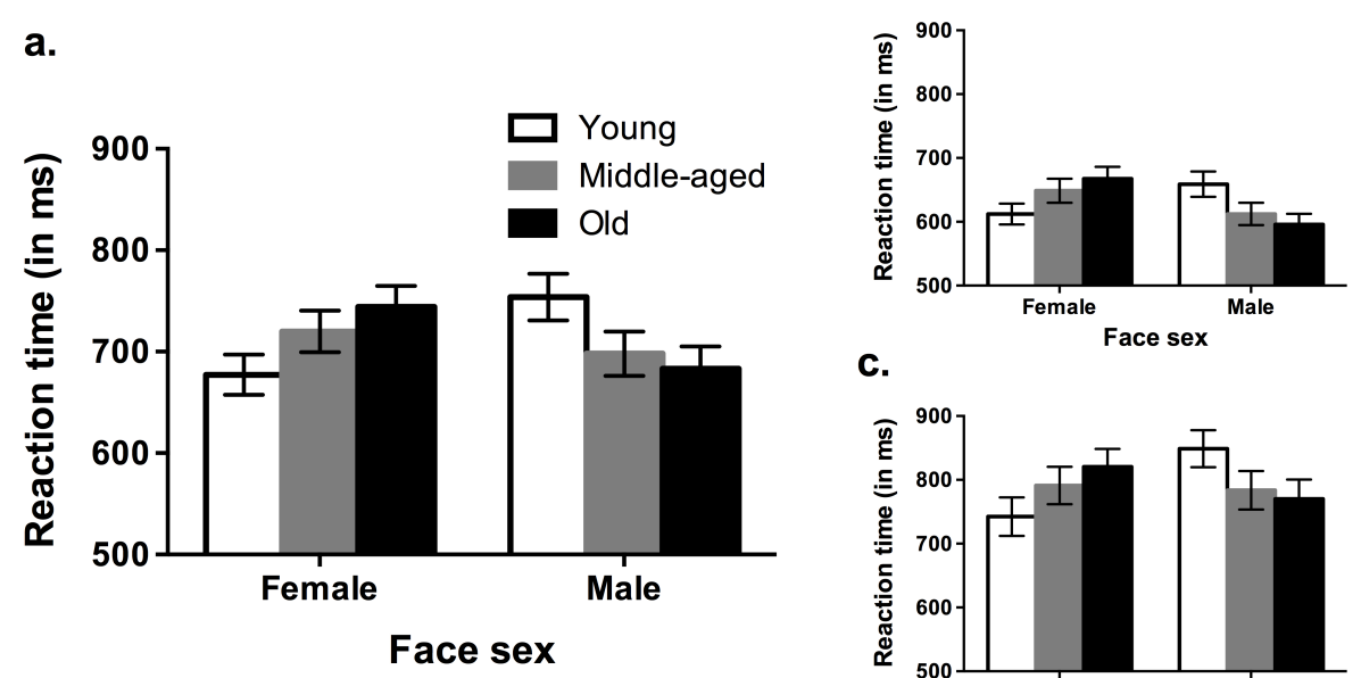

c.

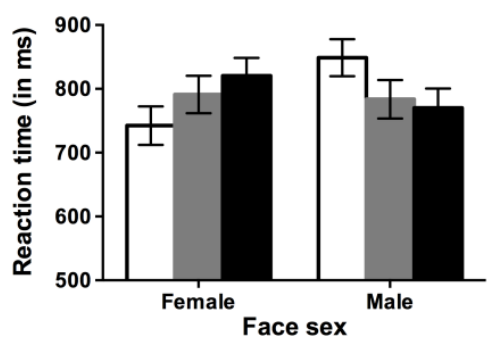

Figure 4 : Results of Experiment 2. a. Opposite effects of age on the latency of sex categorization for female and male faces. b. Effects of face age on latency of sex categorizations observed in young participants. C. Effects of age on latency of sex categorizations observed in older participants. Error bars indicate SEMs. 
Figure 5

Effects of blurring

a.

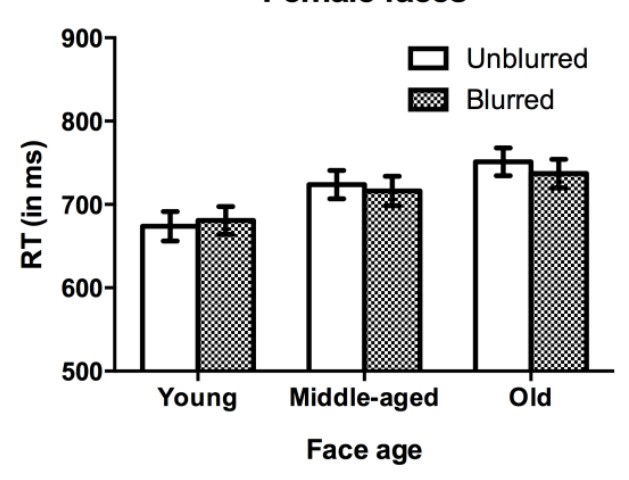

Effects of stimulus orientation

c.

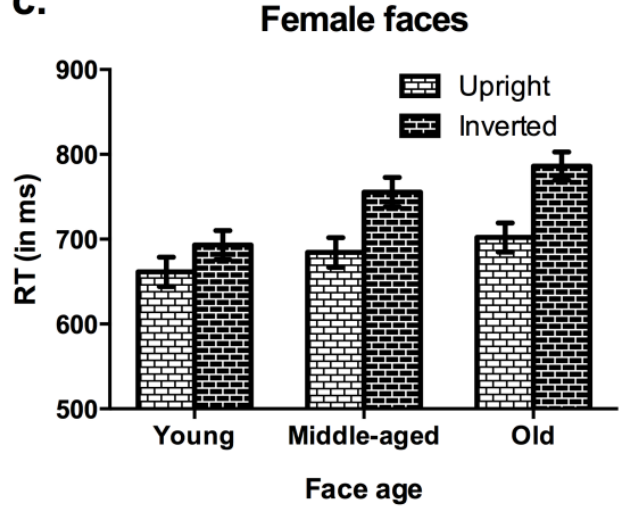

b.

Male faces

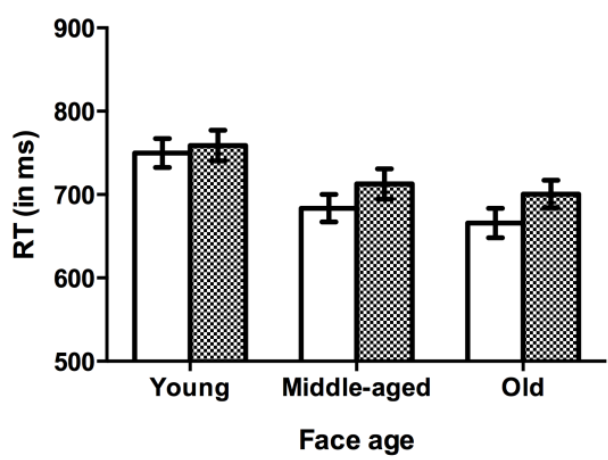

d.

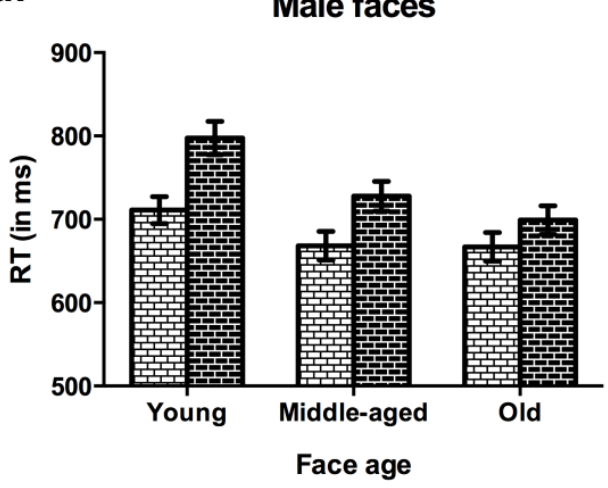

Figure 5 : Results of Experiment 2. a. Effects of blurring on latencies of correct sex categorization of female faces. $b$. Effects of blurring on latencies of correct sex categorization of male faces. c. Effects of stimulus orientation on latencies of correct sex categorization of female faces. d. Effects of stimulus orientation on latencies of correct sex categorization of male faces. Error bars indicate SEMs. 


\section{Figure captions}

Figure 1: Examples of stimuli used in Experiment 1. From left to right: young female face, old female face, young male face, old male face. Please note that copyright limitations prevent depiction of stimulus examples for the middleaged category.

Figure 2: Results of Experiment 1. a. Mean proportion of correct sex categorizations depending on facial age for female and male faces as observed in Experiment 1. B. Mean response latencies of correct sex categorizations depending on facial age for female and male faces as observed in Experiment 1. Error bars indicate SEMs.

Figure 3 : Examples of stimuli used in Experiment 2. Columns (from left to right): young female face, old female face, young male face, old male face. Rows (from top to bottom) : upright unfiltered version, upright filtered version, inverted unfiltered version, inverted filtered version. Please note that copyright limitations prevent depiction of stimulus examples for the middle-aged category.

Figure 4 : Results of Experiment 2. a. Opposite effects of age on the latency of sex categorization for female and male faces. b. Effects of face age on latency of sex categorizations observed in young participants. C. Effects of age on latency of sex categorizations observed in older participants. Error bars indicate SEMs.

Figure 5 : Results of Experiment 2. a. Effects of blurring on latencies of correct sex categorization of female faces. $b$. Effects of blurring on latencies of correct sex categorization of male faces. c. Effects of stimulus orientation on latencies of correct sex categorization of female faces. d. Effects of stimulus orientation on latencies of correct sex categorization of male faces. Error bars indicate SEMs. 